\title{
Benefits from standalone durvalumab treatment in an elderly patient with advanced prostatic sarcoma: a case report
}

\author{
Lili Wang $^{1 \#}$, Lei Wang ${ }^{1 \#}$, Dingkun Hou ${ }^{1}$, Shiqiang Dong ${ }^{1}$, Aixiang Wang ${ }^{2}$, Haitao Wang ${ }^{1}$ \\ ${ }^{1}$ Department of Oncology, The Second Hospital of Tianjin Medical University, Tianjin Institute of Urology, Tianjin, China; ${ }^{2}$ Department of \\ Pathology, The Second Hospital of Tianjin Medical University, Tianjin Institute of Urology, Tianjin, China \\ \#These authors contributed equally to this work. \\ Correspondence to: Haitao Wang. Department of Oncology, The Second Hospital of Tianjin Medical University, Tianjin Institute of Urology, No. 23, \\ Pingjiang Road, Hexi District, Tianjin 300211, China. Email: peterrock2000@126.com.
}

\begin{abstract}
There is a lacking of effective therapeutic strategies in the treatment of advanced prostatic sarcoma with high-frequency microsatellite instability (MSI-H) or mismatch repair deficient (dMMR). In this study, we present the first described a case of advanced MSI-H and dMMR prostatic sarcoma in elderly patients with multiple comorbidities, who received an anti-PD-L1 monoclonal antibody (durvalumab) as the first-line treatment and achieved partial remission (PR) without visible adverse events. A 91-year-old male patient presented with frequent urination and defecation difficulty for over three months, aggravating for ten days. Digital rectal examination showed the prostate gland was $\mathrm{II}^{\circ}$ enlargement and tough with a smooth surface. The MRI showed occupying lesions in the prostate without distant metastasis. Then, the prostate biopsy showed poorly differentiated small round cell malignant tumor and considered prostatic sarcoma. Immunohistochemistry showed MSI-H and dMMR prostatic sarcoma. Durvalumab alone was applied at a cycle of every 21 days (500 mg/day) for 18 months and achieved PR two months since the treatment. During the treatment, we didn't observe rash, immune-related pneumonia, hepatitis, and other adverse events. Also, no recurrence or metastasis was observed until now. Durvalumab is effective and safe in the treatment of advanced MSI-H or dMMR prostatic sarcoma in an elderly patient. It is promising to be an available choice for advanced prostate sarcoma, which is unsuitable for surgery, conventional chemotherapy, and radiotherapy.
\end{abstract}

Keywords: Durvalumab; prostate sarcoma; microsatellite instability (MSI); partial remission (PR); case report

Submitted Mar 21, 2021. Accepted for publication Nov 17, 2021.

doi: $10.21037 /$ tau-21-125

View this article at: https://dx.doi.org/10.21037/tau-21-125

\section{Introduction}

Prostatic sarcoma is a rare and highly invasive malignant tumor of the genitourinary system. Statistically, prostatic sarcoma accounts for $0.7 \%$ of prostate cancer, which can occur at all ages, but prone to middle-aged and older adults with an average age of 61 years (1). Prostatic sarcoma originates in the genital sac. The endoderm tissue can also come from the cricoid muscle. It can be divided into three types according to the cell morphology and pathology $(2,3)$, Type I: myogenic sarcoma: including leiomyosarcoma and rhabdomyosarcoma, Type II: fibrogenic sarcoma: including fibrosarcoma and spindle cell sarcoma, Type III: others including myxosarcoma, liposarcoma, osteosarcoma, and neurogenic sarcoma. The most common types are rhabdomyosarcoma and leiomyosarcoma. Rhabdomyosarcoma is common in children, while leiomyosarcoma is more common in middle-aged and older men (4). The etiology may be related to embryogenesis, malformation, and prostatitis.

The clinical symptoms of prostatic sarcoma are not specific, with progressive dysuria and urinary tract irritation as the first symptoms (5). At present, surgery is one of the essential treatments, while it is unsuitable for older adult patients, especially those with multiple comorbidities (6). High-frequency microsatellite instability (MSI-H)/ mismatch repair deficient (MMR-D) have been identified 
as a marker for immune checkpoint blockade response. This identification is a landmark in treating advanced solid tumors (7). Thus, in recent clinical cancer treatment, detection of MSI-H/dMMR has been regarded as a routine assay in patients with advanced solid tumors (8). Also, MSI-H or dMMR sarcoma is not sensitive to traditional chemotherapy. Therefore, novel and specific therapeutic strategies are in urgent need to improve the therapeutic efficacy of prostatic sarcoma.

Durvalumab is an anti-PD-L1 monoclonal antibody of human immunoglobulin IgG1 $\kappa$, which can block the interaction between PD-L1 and PD-1, CD80 (B7.1) (9-11). Durvalumab in combination with Olaparib was effective in in metastatic castration-resistant prostate cancer with abnormal DNA damage repair, with acceptable toxicity (12). There is no report about the treatment of advanced prostate sarcoma with durvalumab at home and abroad. In this case, we firstly described an elderly patient with advanced MSI-H and $\mathrm{dMMR}$ prostatic sarcoma and multiple comorbidities, who received an anti-PD-L1 monoclonal antibody (durvalumab) as the first-line treatment and achieved partial remission (PR) without visible adverse events. Several characteristics of this case: advanced age, many comorbidities, poor tolerance to conventional radiotherapy and chemotherapy. We present the following article in accordance with the CARE reporting checklist (available at https://dx.doi.org/10.21037/tau-21-125).

\section{Case presentation}

\section{Patient information}

The patient was a 91-year-old male. First arrived outpatient department of our hospital on April 10, 2018, because of the progressive urination and defecation difficulty for over three months, aggravating for ten days.

He has been diagnosed with type II diabetes mellitus for over 20 years, and blood glucose was well controlled (blood glucose fasting: $5.0-6.0 \mathrm{mmol} / \mathrm{L}$, postprandial blood glucose: $8.0-9.0 \mathrm{mmol} / \mathrm{L}$ ) with the treatment of subcutaneous injection of insulin glargine and oral Acarbose. The patient had a history of hypertension and coronary atherosclerotic heart disease for over ten years, which was well controlled by oral simvastatin, betaloc, and aspirin. Also, hepatitis, tuberculosis, and other medical histories were denied.

The patients had a history of surgery; TURP was performed in the treatment of prostate hyperplasia in 2014.
Also, coronary stent implantation was performed in the treatment of coronary heart disease in 2015.

The patient had a smoking history of over 60 years, with 40 cigarettes/day, and alcohol drinking history of over 60 years, $100 \mathrm{~g} /$ day. No family history of cancer was reported.

The study was conducted in accordance with the Declaration of Helsinki (as revised in 2013). Written informed consent was obtained from the patient for publication of this case report and accompanying images. A copy of the written consent is available for review by the editorial office of this journal. The ethics committee of The Second Hospital of Tianjin Medical University approved this study (No.: KY2017k045).

\section{Examination and diagnosis}

Physical examination: heart, lung, and abdomen (-), normal development of external genitalia.

Digital rectal examination: rectum was empty without tumor and wave motion. The prostate gland was $\mathrm{III}^{\circ}$ hyperplasia, tough with a smooth surface, shallow central sulcus without nodule or mass. Also, no blood-stained fingertip.

Laboratory examination: including the blood routine, blood biochemical hepatic and renal functions, electrolyte, myocardial enzymes, cardiac troponins, brain natriuretic peptide, thyroid functions, and ECG were normal.

Tumor markers: including PSA, AFP, CA125, CA199 and CEA.

Chest CT: no obvious abnormality in both lungs.

Color Doppler ultrasound: no obvious abnormality is found in the heart, liver, gallbladder, spleen, pancreas, and kidney.

Pelvic MRI: occupying lesions in the prostate (Figure 1), which breaks through the prostate and invades the rectum. Also, the pelvic cavity shows multiple lymph node metastases.

Prostate biopsy: poorly differentiated small round cell, malignant tumor, prostatic sarcoma (Figure 2).

Immunohistochemistry: vimentin (+), Ki67 $(50 \%+)$, LCA (-), CK wide (-), PSA (-), P504S (-), CGA (-), syn (-), CD117 (-), CD99 (-), PMS2 (-), MLH1 (+), MSH2 (+), MSH6 (+), MMR (-), considered MSI-H and dMMR, prostatic sarcoma

Diagnosis: from the findings above, the patients were diagnosed as advanced MSI-H and dMMR prostatic sarcoma 

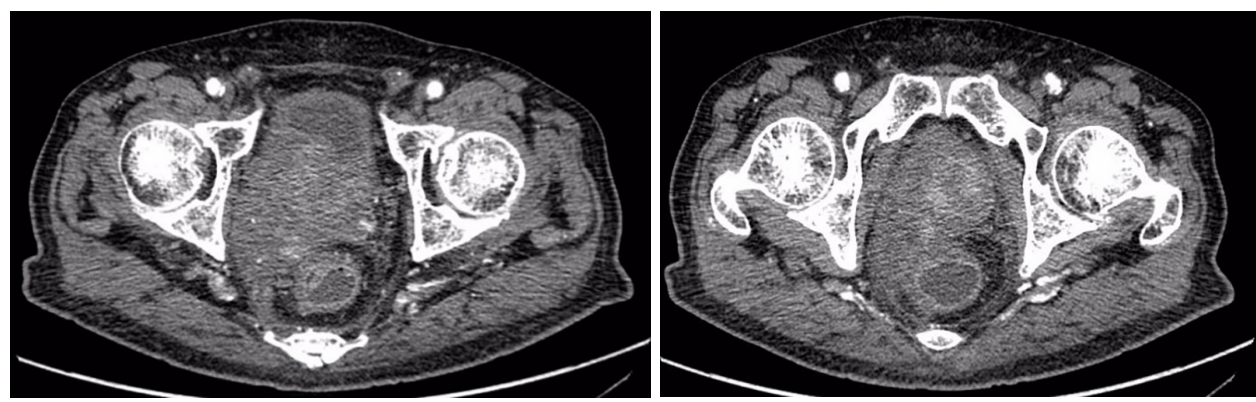

Figure 1 Pelvic MRI. Left: pelvic MRI suggests a space-occupying lesion in the prostate region, locally invading the rectum and seminal vesicle. Right: pelvic MRI suggests a space-occupying lesion in the prostate region, local invasion of rectum and seminal vesicle.

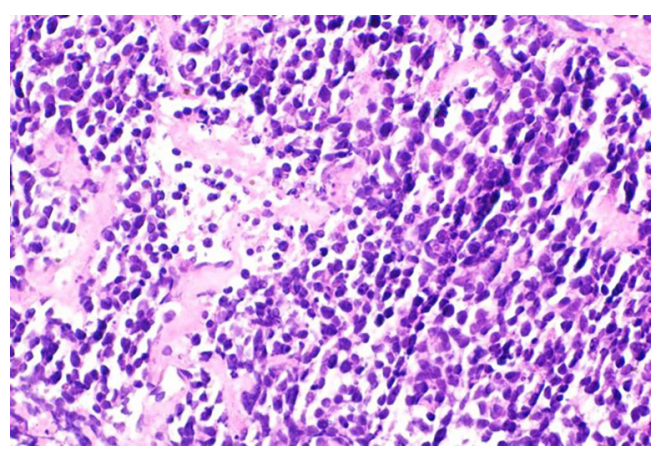

Figure $2 \mathrm{H} \& \mathrm{E}$ stain of the cancer tissue $(400 \times)$.

\section{Treatment}

The patient received the first durvalumab alone treatment since April 27, 2018. Durvalumab was intravenously infused once every 21 days at a dose of $500 \mathrm{mg}$ for 18 months. Also, the treatment strategy was continued until disease progression or unacceptable toxicity.

During treatment, blood routine, hepatic and renal functions, electrolyte, myocardial enzyme, cardiac troponin, BNP, thyroid function, and ECG are monitored weekly. Also, chest CT, Pelvic MRI, color Doppler ultrasound in the heart and abdomen were examined every two cycles.

The therapeutic efficacy was tested two months since the treatment started.

\section{Treatment efficiency evaluation standards and adverse events}

Primary efficacy measures are the progression-free survival (PFS), defined as the time from date of the first dose to the earliest occurrence of disease progression or death from any cause.
Secondary efficacy measures: according to the RECIST evaluation standard, the therapeutic efficacy was tested. Complete remission (CR) was CT or endoscopy, which lasted at least four weeks, could not detect any target lesions. PR was referred as a $>50 \%$ reduction on the sum of products of the two longest perpendicular diameters of target lesions. Stable disease (SD) was defined to as a $\leq 50 \%$ decrease or a $\leq 25 \%$ increase in the sum of products of the two longest perpendicular diameters of target lesions. Progressive disease (PD) was defined to as a $>20 \%$ increase on the sum of products of the two longest perpendicular diameters, at least one measurable or assessable lesion, or as the appearance of a new lesion. Response rate (RR) was defined to as $\mathrm{CR}+\mathrm{PR}$, disease control rate (DCR) is CR + $\mathrm{PR}+\mathrm{SD}$.

Adverse events: the treatment associated side events were evaluated by the National Cancer Institute-Common Toxicity Criteria (NCI-CTC, version 3.0), which graded the adverse events into four levels (I to IV). Treatment was put off when the grade III or above adverse events occurred. Re-application was applied at a $25 \%$ dose reduction upon adverse events reduced to grade II. The treatment could be discontinued when the grade III or above side adverse events cannot be controlled even with a reduced dose two times.

\section{Efficacy and adverse event evaluation}

The patient finished an 18-month treatment with proper compliance and tolerance. During the treatment, the symptoms of urination and defecation were significantly improved. Also, two months after treatment, the patients achieved PR (Figure 3).

During the treatment, we didn't observe rash, immunerelated pneumonia, hepatitis, and other adverse events, and 

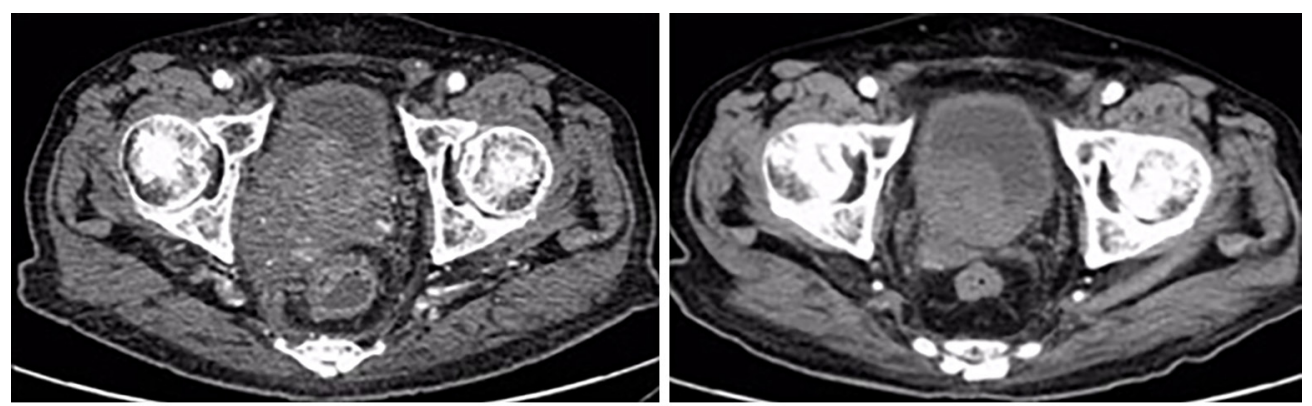

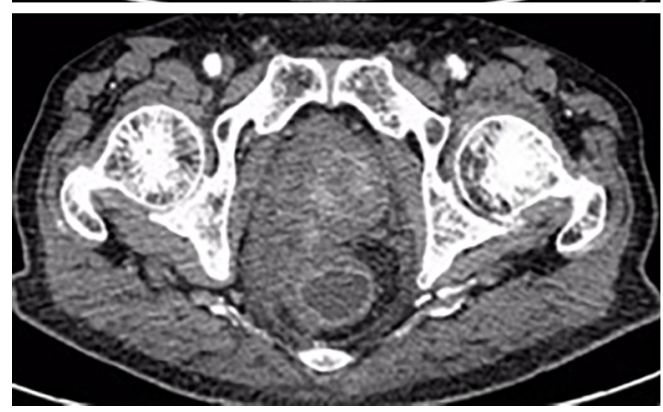

Before the treatment

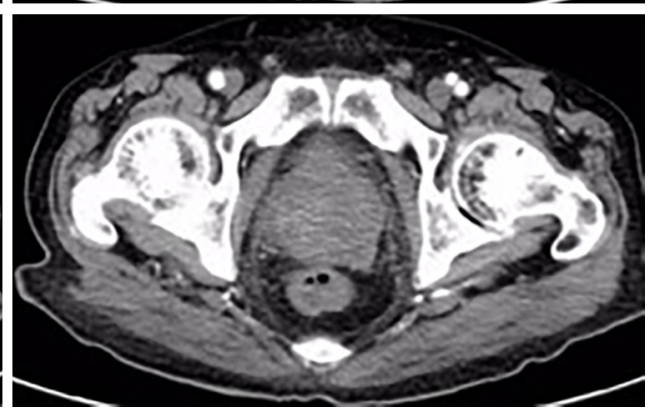

After the treatment

Figure 3 Pelvic MRI before and after the treatment. The two images on the left represent the pelvic state of the patient before treatment, with a large space-occupying lesion in the prostate and local invasion of the rectum. The two images on the right show a significant reduction in prostate lesions and reduced rectal compression after immunotherapy.

the PS score was 0.

Also, pelvic MRI was examined every two months, no new metastasis was observed, and the local tumor size was significantly reduced than before, and no recurrence or metastasis was observed.

\section{Discussion}

Prostatic sarcoma is a malignant tumor of the genitourinary system, with rapid progress and poor prognosis. The specific therapeutic strategy depends on the patient's age, health status, tumor size, disease progress. At present, comprehensive therapy after radical prostatectomy is recommended as the primary therapeutic strategy in the treatment of prostatic sarcoma (13-15). At the same time, it is not available for elderly patients, especially those with multiple comorbidities.

Recent studies have confirmed the relationship between immune checkpoints and the progression of malignant tumors, and the efficacy of immunosuppressive checkpoint inhibitors for various malignant tumors (16-18). MSI is mainly induced by $\mathrm{dMMR}$, which could accumulate elevated levels of mutation and produce new antigens, leading to an increased sensitivity to PD-1/PD-L1. Exon sequencing showed that more somatic mutations occurred in MSI tumors, and the somatic mutation rate was positively related to PFS, suggesting dMMR-based MSI tumors may benefit from immunotherapy.

The results of clinical trials, including keynote-012, keynote-016, keynote-028, keynote-158, and keynote-164, showed that the remission rate of PD-1 antibody pembrolizumab was $39.6 \%$ in the treatment of advanced solid tumors with MSI-H. From these results from clinical trials, pembrolizumab was approved in the treatment of solid tumors with MSI-H on May 23, 2017, regardless of tumor type and location (7).

Durvalumab is a selective, high affinity, PD-L1 monoclonal antibody. Durvalumab blocks the binding sites of PD-L1 with PD-1 and CD80. Thus, T cells could recognize and kill tumor cells (9-11). In addition to inhibiting the PD-1-PD-L1 pathway, PD-L1 mAb can also help fully activate $\mathrm{T}$ cell functions and cytokine production by blocking the co-inhibitory functions of B7.1 and PDL1. So, in theory, PD-L1 monoclonal antibody is stronger. PD1 monoclonal antibody can block the binding of PDL1 and PD-L2 to PD-1 at the same time, while PD-L1 monoclonal antibody only blocks the binding of PD-L1 to PD-1 and retains PD-L2 of macrophages, which can 
effectively avoid interstitial pneumonia (breaking PDL2 function can lead to abnormal activation of RGMb and cause pneumonia) and other side effects. In May 2017, the FDA approved the application of durvalumab in urothelial cancer patients with advance locally or metastasis during chemotherapy with platinum drugs or neoadjuvant chemotherapy with platinum drugs within 12 months. On February 19, 2018, durvalumab was approved in the treatment of stage III NSCLC in over 40 countries or regions in the world. A multi-cohort clinical study showed that the total remission rate of durvalumab combined with tremelimumab in the treatment of advanced solid tumors was $41 \%$. The survival period was 26 months.

The efficacy of durvalumab in the treatment of prostatic sarcoma has not been well investigated. Even though durvalumab alone or combined with tremelimumab has been carried out in cumulative clinical trials in sarcomas, including Nexis (NCT03116529) (19), (NCT02815995) (20), (NCT03317457) (21), the efficacy evaluation has not been reported. In this study, we report a case of advanced MSI-Hand dMMR prostatic sarcoma in an elderly patient with multiple comorbidities. There is no opportunity for surgery, no sensitivity to conventional chemotherapy and radiotherapy, and no particular and effective treatment strategy. While the patient benefited from the immunotherapy with PD-L1 mAb durvalumab alone without visible adverse events, showing that durvalumab is safe and effective in the treatment of advanced MSI-H or dMMR prostatic sarcoma in the elderly patient, might be an available choice for those patients who are not suitable for surgery or conventional therapeutic strategies.

To our knowledge, this is the first described in a case of advanced MSI-H, and dMMR prostate sarcoma benefited from immunotherapy with durvalumab alone. However, the therapeutic efficacy from one case cannot reflect the and the definitive results. Also, the efficacy of durvalumab in the treatment of sarcoma from the above clinical trial will shed light on optimizing the current therapeutic strategies for prostatic sarcoma. The patient in this case was satisfied with the treatments.

\section{Conclusions}

Durvalumab is effective and safe in the treatment of advanced MSI-H or DMMR prostatic sarcoma in an elderly patient. It is promising to be an available choice for advanced prostate sarcoma, which is unsuitable for surgery, conventional chemotherapy, and radiotherapy.

\section{Acknowledgments}

Funding: This study was supported in part by the National Natural Science Foundation (81572543) and Tianjin Science \& Technology Plan Project (7ZXMFSY00040).

\section{Footnote}

Reporting Checklist: The authors have completed the CARE reporting checklist. Available at https://dx.doi. org/10.21037/tau-21-125

Conflicts of Interest: All authors have completed the ICMJE uniform disclosure form (available at https://dx.doi. org/10.21037/tau-21-125). The authors have no conflicts of interest to declare.

Ethical Statement: The authors are accountable for all aspects of the work in ensuring that questions related to the accuracy or integrity of any part of the work are appropriately investigated and resolved. The study was conducted in accordance with the Declaration of Helsinki (as revised in 2013). Written informed consent was obtained from the patient for publication of this case report and accompanying images. A copy of the written consent is available for review by the editorial office of this journal. The ethics committee of The Second Hospital of Tianjin Medical University approved this study (No.: KY2017k045).

Open Access Statement: This is an Open Access article distributed in accordance with the Creative Commons Attribution-NonCommercial-NoDerivs 4.0 International License (CC BY-NC-ND 4.0), which permits the noncommercial replication and distribution of the article with the strict proviso that no changes or edits are made and the original work is properly cited (including links to both the formal publication through the relevant DOI and the license). See: https://creativecommons.org/licenses/by-nc-nd/4.0/.

\section{References}

1. Hicks N, Gurung PM, Deshmukh N, et al. Primary prostate sarcoma: how to manage following diagnosis at transurethral resection. J Surg Case Rep 2016;2016:rjw065.

2. Welz S, Hehr T, Lamprecht U, et al. Thermoradiotherapy of the chest wall in locally advanced or recurrent breast cancer with marginal resection. Int J Hyperthermia 2005;21:159-67. 
3. Ohguri T, Imada H, Kato F, et al. Radiotherapy with 8 $\mathrm{MHz}$ radiofrequency-capacitive regional hyperthermia for pain relief of unresectable and recurrent colorectal cancer. Int J Hyperthermia 2006;22:1-14.

4. Wang X, Liu L, Tang H, et al. Twenty-five cases of adult prostate sarcoma treated at a high-volume institution from 1989 to 2009. Urology 2013;82:160-5.

5. Abbas SM, Hill AG. Prostatic sarcoma after treatment of rectal cancer. World J Surg Oncol 2007;5:82.

6. De Bari B, Stish B, Ball MW, et al. Adult prostatic sarcoma: A contemporary multicenter Rare Cancer Network study. Prostate 2017;77:1160-6.

7. Le DT, Uram JN, Wang H, et al. PD-1 Blockade in Tumors with Mismatch-Repair Deficiency. N Engl J Med 2015;372:2509-20.

8. Latham A, Srinivasan P, Kemel Y, et al. Microsatellite Instability Is Associated With the Presence of Lynch Syndrome Pan-Cancer. J Clin Oncol 2019;37:286-95.

9. Stewart R, Morrow M, Hammond SA, et al. Identification and Characterization of MEDI4736, an Antagonistic Anti-PD-L1 Monoclonal Antibody. Cancer Immunol Res 2015;3:1052-62.

10. Pardoll DM. The blockade of immune checkpoints in cancer immunotherapy. Nat Rev Cancer 2012;12:252-64.

11. Postow MA, Callahan MK, Wolchok JD. Immune Checkpoint Blockade in Cancer Therapy. J Clin Oncol 2015;33:1974-82.

12. Karzai F, VanderWeele D, Madan RA, et al. Activity of durvalumab plus olaparib in metastatic castration-resistant prostate cancer in men with and without DNA damage repair mutations. J Immunother Cancer 2018;6:141.

13. Murakami Y, Tabata K, Sugita A, et al. Multidisciplinary treatment including systemic chemotherapy for a malignant phyllodes tumour of the prostate. Can Urol Assoc J 2014;8:E263-5.

Cite this article as: Wang L, Wang L, Hou D, Dong S, Wang A, Wang H. Benefits from standalone durvalumab treatment in an elderly patient with advanced prostatic sarcoma: a case report. Transl Androl Urol 2021;10(11):4298-4303. doi: 10.21037/tau-21-125
14. Musser JE, Assel M, Mashni JW, et al. Adult prostate sarcoma: the Memorial Sloan Kettering experience. Urology 2014;84:624-8.

15. Smith MJ, Ridgway PF, Catton CN, et al. Combined management of retroperitoneal sarcoma with dose intensification radiotherapy and resection: longterm results of a prospective trial. Radiother Oncol 2014;110:165-71.

16. Sharma P, Allison JP. The future of immune checkpoint therapy. Science 2015;348:56-61.

17. Lerrer S, Mor A. Immune checkpoint inhibitors and the shared epitope theory: from hy-pothesis to practice. Transl Cancer Res 2019;8:S625-7.

18. Santoni M, Montironi R, Battelli N. Immune Checkpoint Blockade in Advanced Renal-Cell Carcinoma. N Engl J Med 2018;379:91-2.

19. Somaiah N, Conley AP, Lin H, et al. A phase II multi-arm study to test the efficacy of durvalumab and tremelimumab in multiple sarcoma subtypes (abstract). 22nd Connective Tissue Oncology Society (CTOS) Meeting: Maui, Hawaii; 2017:2804799.

20. Neoadjuvant Durvalumab and Tremelimumab Plus Radiation for High Risk Soft-Tissue Sarcoma (NEXIS). University of Maryland. 2017. Available online: https:// clinicaltrials.gov/ct2/show/NCT03116529. Accessed October 212017.

21. Durvalumab and Tremelimumab Compared to Doxorubicin in Patients With Advanced or Metastatic Soft Tissue Sarcoma. Available online: https://www.drugbank. ca/drugs/DB00997/clinical_trials?conditions=DBCOND0 $037580 \%$ 2CDBCOND0028931\&phase $=2 \&$ purpose $=$ treat ment\&status=recruiting

(English Language Editor: J. Chapnick) 